\title{
Declínio de atividades instrumentais de vida diária associado à perda de força de preensão palmar em idosos internados em enfermaria geriátrica
}

\section{Decline in instrumental activities of daily living associated with loss of hand grip strength in elderly patients hospitalized in geriatric ward}

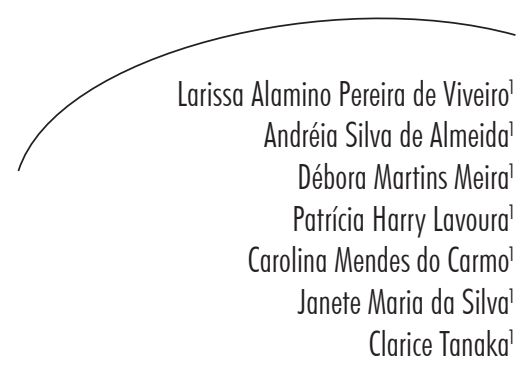

Resumo

Objetivo: Verificar a associação entre a capacidade de executar as atividades instrumentais de vida diária (AIVDs) e a força de preensão palmar em idosos sem comprometimento cognitivo internados em enfermaria geriátrica. Métodos: Estudo transversal. Foram incluídos pacientes idosos, com idade igual ou superior a 60 anos, de ambos os gêneros, admitidos em enfermaria de Geriatria. Foram excluídos indivíduos com doenças neurológicas, osteomusculares agudas e respiratórias exacerbadas; com indicação médica de repouso ou com uso de algum aparato clínico, bem como aqueles com déficits cognitivos e/ou delirium. Os dados foram coletados do prontuário e foi realizada a avaliação da capacidade de realizar atividades instrumentais de vida diária, por meio da Escala de Lawton, e a avaliação da força de preensão palmar, por meio da dinamometria do membro superior dominante. Os dados foram descritos em média e desvio- padrão. O teste de correlação de Pearson foi aplicado para verificar associação entre a capacidade de realizar AIVDs e a força de preensão palmar. Resultados: Participaram do estudo 12 idosos (75\% mulheres) com idade média de 80,67£6,35 anos. As médias da força de preensão palmar e pontuação total da Escala de Lawton foram

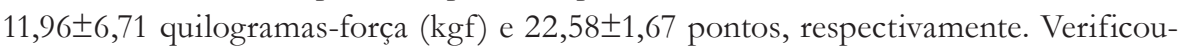
se associação moderada e positiva entre a força de preensão palmar e a pontuação na Escala de Lawton $(r=0,640 ; \mathrm{p}=0,025)$. Conclusão: A capacidade de realizar AIVDs e a força de preensão palmar apresentaram associação na amostra estudada.

\section{Abstract}

Objective: To verify the association between the ability to perform instrumental activities of daily living (IADL) and handgrip strength in hospitalized geriatric patients without cognitive impairment. Methods: Cross-sectional study. Elderly patients aged over 60 years of both genders admitted to geriatric patients ward were included. On the other hand, elderly with neurological, musculoskeletal and respiratory acute exacerbated diseases; individuals with medical indication of rest or use of clinical apparatus, cognitive impairment and/or delirium were excluded. Data were collected from medical records.

Serviço de Fisioterapia, Instituto Central do Hospital das Clínicas, Faculdade de Medicina. Universidade de São Paulo. São Paulo, SP, Brasil.

Palavras-chave: Idoso. Idoso Fragilizado. Saúde do Idoso. Hospitalização. Atividades Cotidianas. 
The ability to perform IADL was assessed by Lawton Scale and the handgrip strength was measured using handheld dynamometry on the dominant upper limb. A descriptive analysis with mean and standard deviation was carried out. The Pearson correlation test was applied to verify association between the total score of Lawton Scale and handgrip strength. Results: The study included 12 patients (75\% women) whose mean age were $80.67 \pm 6.35$ years. The mean of handgrip strength and total Lawton Scale score were $11.96 \pm 6.71 \mathrm{Kgf}$ and $22.58 \pm 1.67$ points, respectively. A moderate positive association between handgrip strength and Lawton Scale score $(r=0.640 ; p=0.025)$ was found. Conclusion: The handgrip strength and IADLs were associated in this study population.
Key words: Elderly. Frail Elderly. Health of the Elderly. Hospitalization. Activities of Daily Living.

\section{INTRODUÇÃO}

O envelhecimento populacional se traduz em maior prevalência de comorbidades e mais incapacidade, o que pode culminar em maior risco de internação hospitalar da população idosa. Quando comparados a outras faixas etárias, os idosos apresentam maior número de internações e tempo de ocupação dos leitos. ${ }^{1}$

A internação hospitalar é considerada de grande risco às pessoas idosas, à medida que estas são mais suscetíveis a complicações provocadas pelo repouso prolongado no leito, ${ }^{2,3}$ à ocorrência de fragilidade ${ }^{4} \mathrm{e}$ ao declínio funcional após a hospitalização., ${ }^{5,6} \mathrm{~A}$ fragilidade pode ser definida como um estado dinâmico que afeta um indivíduo nos aspectos físico, psíquico e social, causando redução de sua reserva fisiológica e declínio da capacidade funcional. ${ }^{7}$

Pessoas idosas com declínio funcional possuem risco de mortalidade, ${ }^{8}$ necessidade de cuidados e elevado custo para os serviços de saúde. ${ }^{9-11} \mathrm{O}$ declínio da capacidade funcional no envelhecimento se traduz na dificuldade em realizar as atividades básicas de vida diária (ABVDs) - banhar-se, vestir-se, alimentar-se, andar, transferir-se, ter controle esfincteriano vesical e fecal - e atividades instrumentais de vida diária (AIVDs) - cuidar das finanças, usar o telefone, controlar medicamentos, realizar compras, entre outras.

Sabe-se na literatura que a fraqueza muscular, ${ }^{12}$ fatores como estado civil (ser viúvo ou solteiro) e ter uma autopercepção de saúde ruim são determinantes de dependência para realização de ABVDs. Em contrapartida, o prejuízo da memória verbal, déficit cognitivo, idade mais avançada, depressão, número de comorbidades, redução da acuidade visual e amputação de membro podem ser determinantes de dependência para realização de AIVDs. ${ }^{13,14}$

Não está claro na literatura se a perda de força muscular está associada à dependência para realização das AIVDs em idosos sem déficit cognitivo. A verificação da força muscular na pessoa idosa faz parte da avaliação geriátrica ampla, é simples, fácil de ser realizada e pode ser estimada por meio da força de preensão palmar verificada com o uso de dinamometria de mão. ${ }^{15,16}$

Considerando essa lacuna na literatura, o objetivo deste estudo foi verificar a associação entre a capacidade de executar as atividades instrumentais de vida diária e a força de preensão palmar em idosos sem comprometimento cognitivo internados em enfermaria geriátrica.

\section{METODOLOGIA}

Trata-se de estudo transversal, aprovado pelo Comitê de Ética em Pesquisa do Hospital das Clínicas da Faculdade de Medicina da Universidade de São Paulo (HCFMUSP), sob o $n^{\circ} 0740 / 10$, realizado na enfermaria de Geriatria de um hospital público terciário de grande porte, entre os meses de setembro e outubro de 2011. Todos os participantes assinaram o Termo de Consentimento Livre e Esclarecido. O estudo não apresentou cálculo amostral, pois se trata de um estudo-piloto pertencente a um estudo 
longitudinal que objetivou verificar o desfecho funcional do idoso após a internação hospitalar.

Os critérios de inclusão foram: pacientes idosos, com idade igual ou superior a 60 anos, de ambos os gêneros e admitidos na enfermaria de Geriatria do HCFMUSP. Critérios de exclusão: pacientes com doenças neurológicas, doenças osteomusculares agudas ou deformidades de membros superiores e mãos, que impediam o idoso de realizar os testes propostos e lhe causassem dor (pontuação na Escala Analógica Visual de Dor - EVA $\geq 8),{ }^{17}$ doenças respiratórias exacerbadas (pontuação na Escala de Borg modificada para sensação de dispneia $\geq 7),{ }^{18}$ indivíduos com indicação médica de repouso e/ou com uso de algum aparato clínico (como drenos ou cateteres venoso central com infusão contínua de medicação); pacientes com déficits cognitivos (pontuação do Miniexame do Estado Mental - MEEM (segundo a escolaridade) ${ }^{19} \mathrm{e} / \mathrm{ou}$ que estivessem em delirium (verificado pela equipe médica como rotina da enfermaria por meio da escala Confusion Assessment Method - CAM ${ }^{20}$ ).

No período de coleta do estudo, 36 pacientes idosos (27 mulheres e nove homens) foram internados na enfermaria de Geriatria. Destes, 16 foram excluídos por doença neurológica, inclusive demência; três por doença osteomuscular exacerbada; dois por déficit cognitivo; dois por aparatos clínicos e um por delirium, constituindo uma amostra de 12 pacientes idosos elegíveis e que aceitaram participar do estudo.

O recrutamento dos pacientes foi feito diariamente por meio de uma triagem na enfermaria, a fim de identificar aqueles que atendiam aos critérios de elegibilidade. A coleta do protocolo proposto consistiu em duas etapas. Primeiro, foram coletados dos prontuários os dados sociodemográficos, como idade, gênero, presença e número de comorbidades e motivo da internação hospitalar.

Em seguida, foi realizada a avaliação das AIVDs e da força de preensão palmar. A avaliação da capacidade de execução das AIVDs foi efetuada por meio da aplicação da Escala de Lawton. ${ }^{21}$ Esta avalia oito tarefas necessárias para a vida independente na comunidade: usar o telefone, utilizar transporte, fazer compras, preparar refeições, arrumar a casa, fazer trabalhos manuais domésticos, manusear medicamentos e cuidar das finanças. Para cada tarefa, há três possibilidades de resposta com valores de pontuação de 1 a 3 (1 - dependência; 2 - capacidade de realizar a tarefa com ajuda; e 3 - independência). A pontuação final é alcançada pela soma de pontos dos oito domínios e varia de 8 a 24, de tal forma que quanto maior for a pontuação, mais independente será o indivíduo para executar a atividade. ${ }^{22}$ Cada idoso foi questionado domínio a domínio, e foi dado como tempo de referência para avaliação das AIVDs o último mês antes da internação.

A força de preensão palmar foi mensurada por meio de um dinamômetro palmar hidráulico analógico Jamar ${ }^{\circledR}$. Foram seguidas as recomendações da American Society of Hand Therapists ${ }^{23}$ para padronização da medida realizada. $O$ indivíduo idoso permaneceu sentado em uma cadeira padronizada (sem apoio para antebraços), com a coluna ereta, flexão de joelhos a $90^{\circ}$, ombro posicionado próximo ao corpo e rotação neutra, cotovelo fletido a $90^{\circ}$ e antebraço e punho em posição neutra. A mão dominante do participante manteve-se no dinamômetro, enquanto este foi sustentado pelo avaliador. Foi realizado o comando de "aperte o aparelho o mais fortemente que o(a) senhor(a) puder" e repetido por três vezes, sendo considerada a maior medida.

A descrição das variáveis quantitativas, dados sociodemográficos (idade, gênero, número de comorbidades e motivos de internação) e escores dos instrumentos de avaliação (Escala de Lawton e Força de Preensão Palmar) foram apresentados por meio da média e desvio-padrão, ou porcentagem, quando cabível. As variáveis foram testadas quanto a suas normalidades pelo teste de Shapiro-Wilk. A associação entre a força de preensão palmar e as AIVDs foi verificada por meio do teste de correlação de Pearson. A versão 19 do programa estatístico Statistical Package for the Social Sciences para Windows ${ }^{\circledR}$ (SPSS Inc., Chicago, Illinois) foi utilizada para realização da análise e construção do gráfico. O nível de significância adotado foi de $5 \%(p<0,05)$. 


\section{RESULTADOS}

A amostra foi constituída de 12 pacientes idosos (75\% de mulheres), com média de idade de $80,67 \pm 6,35$ anos e número médio de 5,92 22,31 comorbidades, cuja principal causa de admissão na enfermaria de Geriatria foi a investigação de distúrbios gastrointestinais (58\%). A tabela 1 apresenta os dados sociodemográficos (gênero, idade, número de comorbidades e motivos de internação) e as variáveis de interesse do estudo (força de preensão palmar e escore da Escala de Lawton) nos idosos avaliados.

A média da força de preensão palmar e o escore total da Escala de Lawton foram, respectivamente, $12 \pm 6,7 \mathrm{kgf}$ e $22,6 \pm 1,7$ pontos. Usar o telefone, arrumar a casa e manusear medicamentos foram os únicos domínios em que todas as pessoas idosas avaliadas atingiram pontuação máxima na Escala de Lawton.

Tabela 1. Dados sociodemográficos, força de preensão palmar e capacidade de realizar atividades instrumentais de vida diária em pessoas idosas. São Paulo-SP, 2011.

\begin{tabular}{|c|c|c|}
\hline \multicolumn{2}{|l|}{ Dados } & Valores \\
\hline \multicolumn{2}{|l|}{ Idade (anos)* } & $80,67 \pm 6,35$ \\
\hline \multicolumn{3}{|l|}{ Gênero } \\
\hline \multicolumn{2}{|l|}{ Mulheres } & $9(75 \%)$ \\
\hline \multicolumn{2}{|l|}{ Homens } & $3(25 \%)$ \\
\hline \multicolumn{2}{|l|}{ Número de comorbidades* } & $5,92 \pm 2,31$ \\
\hline \multicolumn{3}{|l|}{ Motivos de internação } \\
\hline \multicolumn{2}{|c|}{ Investigação de distúrbios do sistema gastrointestinal } & $7(58 \%)$ \\
\hline \multicolumn{2}{|l|}{ Complicações metabólicas e/ou renais } & $3(25 \%)$ \\
\hline \multicolumn{2}{|l|}{ Difunções respiratórias e/ou cardíacas } & $2(17 \%)$ \\
\hline \multicolumn{2}{|l|}{ Força de preensão palmar (kgf)* } & $12 \pm 6,71$ \\
\hline \multicolumn{2}{|l|}{ Escore total da Escala de Lawton (pontos)* } & $22,58 \pm 1,67$ \\
\hline \multirow{8}{*}{$\begin{array}{l}\text { Domínios avaliados na Escala de Lawton } \\
\text { (pontos)* }\end{array}$} & Usar o telefone & $3,0 \pm 0$ \\
\hline & Utilizar transporte & $2,5 \pm 0,80$ \\
\hline & Fazer compras & $2,42 \pm 0,79$ \\
\hline & Preparar refeições & $2,92 \pm 0,29$ \\
\hline & Arrumar a casa & $3,0 \pm 0$ \\
\hline & Fazer trabalhos manuais & $2,92 \pm 0,29$ \\
\hline & Manusear medicamentos & $3,0 \pm 0$ \\
\hline & Cuidar das finanças & $2,83 \pm 0,58$ \\
\hline
\end{tabular}

${ }^{*} \pm \underline{d p}=$ média \pm desvio-padrão. 
A figura 1 apresenta a distribuição de todos os indivíduos estudados quanto à força de preensão palmar e o escore total da Escala de Lawton.

A tabela 2 apresenta os coeficientes de Pearson entre idade, escore total e de cada domínio da Escala de Lawton; e a força de preensão palmar da mão dominante da amostra investigada. Foi encontrada associação moderada e positiva entre o escore total da Escala de Lawton e a força de preensão palmar $(r=0,640 ; p=0,025)$. Somente o domínio que diz respeito a realizar compras apresentou associação forte e negativa à força de preensão palmar ( $r=0,840 ; \mathrm{p}=0,001)$. Não está descrito na tabela 2, mas idade e escore total da Escala de Lawton apresentaram associação moderada e negativa $(r=-0,603 ; p=0,03)$.

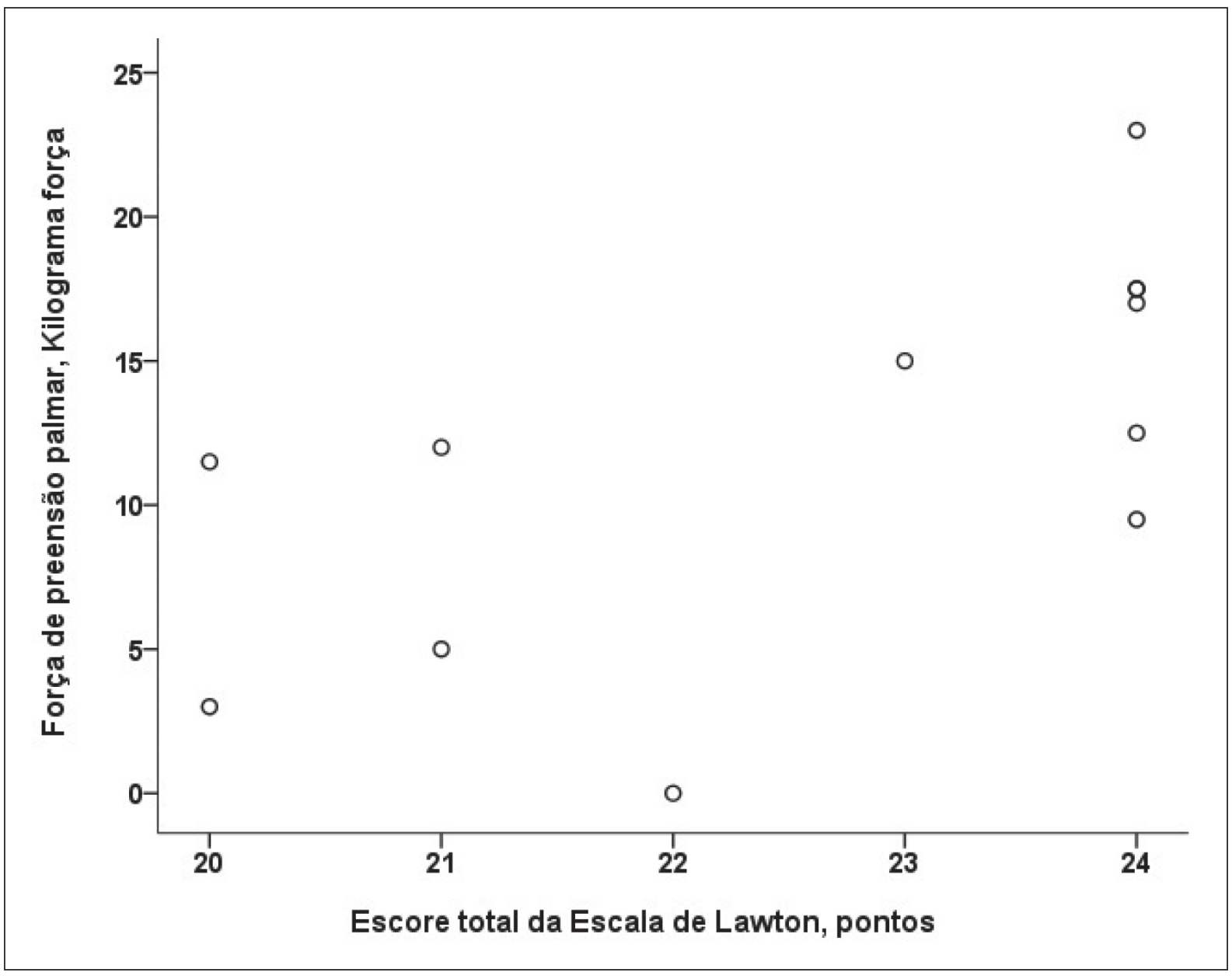

Figura 1. Distribuição das pessoas idosas quanto à força de preensão palmar e escore total da Escala de Lawton. São Paulo-SP, 2011. 
Tabela 2. Coeficientes de correlação de Pearson entre a força de preensão palmar e a capacidade de realização de atividades instrumentais de vida diária em pessoas idosas. São Paulo-SP, 2011.

\begin{tabular}{clcc}
\hline & & Força de preensão palmar da mão dominante \\
\hline \multirow{2}{*}{$\begin{array}{c}\text { Idade (anos) } \\
\text { Escore total da Escala de Lawton (pontos) }\end{array}$} & $\mathrm{r}$ & $\mathrm{p}$ \\
& & $-0,500$ & 0,10 \\
& Usar telefone & 0,640 & $0,02^{\mathrm{a}}$ \\
& Ir a lugares distantes & $*$ & $*$ \\
Domínios & Fazer compras & 0,081 & 0,80 \\
avaliados na & Preparar as refeições & 0,840 & $0,001^{\mathrm{a}}$ \\
Lawton & Arrumar a casa & 0,326 & 0,30 \\
& Realizar trabalhos manuais & $*$ & $*$ \\
& Administrar os medicamentos & 0,021 & 0,95 \\
& Cuidar das finanças & $*$ & 0,17 \\
\hline
\end{tabular}

r= coeficiente de correlação; *não foi possível o cálculo do coeficiente de correlação e p, pois todos os indivíduos apresentaram valor máximo de pontuação no domínio analisado; ${ }^{a}$ significância estatística $(\mathrm{p}<0,05)$.

\section{DISCUSSÃO}

O achado mais relevante do estudo foi a associação moderada e positiva entre a força de preensão palmar e a pontuação total da Escala de Lawton $(r=0,640 ; p=0,025)$. Poucos estudos têm se proposto a verificar o impacto ou associação da força muscular ao declínio da capacidade de executar as AIVDs, pois a perda de força muscular tem sido associada, principalmente, ao prejuízo funcional para realização das ABVDs, enquanto o déficit cognitivo foi encontrado como um dos principais determinantes da capacidade de executar as AIVDs. ${ }^{14}$

Na literatura, alguns autores mencionaram que a medida força muscular, seja por dinamometria ou por força muscular de quadríceps, é a melhor medida associada à limitação funcional e à incapacidade para realização de AIVDs. ${ }^{12,14}$ Hairi et al., ${ }^{12}$ em 2010, realizaram estudo com 1.755 homens idosos e, ao avaliarem a capacidade de realização de ABVDs e AIVDs, dinamometria, força, massa e qualidade muscular do músculo quadríceps, verificaram associação entre a força de preensão palmar e a capacidade de realizar AIVDs. ${ }^{12}$ Embora neste estudo a amostra tenha sido composta somente pelo gênero masculino, os achados corroboram o encontrado na presente pesquisa.

A capacidade de realizar compras foi a única atividade que apresentou associação forte e negativa à força de preensão palmar. A diminuição da força de preensão palmar pode afetar diversas atividades instrumentais de vida diária ${ }^{24}$, à medida que as mãos são utilizadas para executar todas as atividades, como manusear o telefone e medicamentos, carregar sacolas durante as compras, preparar refeições e fazer trabalhos manuais.

Além disso, é importante salientar que a idade média da amostra deste estudo foi de 80 anos e composta, em sua maioria, por mulheres (75\%), o que se assemelha aos estudos de Dutra 
et al. ${ }^{25}$ e Estrella et al., ${ }^{26}$ nos quais as amostras apresentaram, respectivamente, $71,9 \%$ e $12,5 \%$ de mulheres com maior risco de internação. Apesar de o gênero feminino e a idade mais avançada serem fatores reconhecidamente associados a dificuldade na realização de AIVDs, ${ }^{13}$ a média do total de pontos do escore de Lawton foi de 22,58 pontos. A figura 1 mostra que oito $(66,6 \%)$ das 12 pessoas idosas estudadas apresentaram pontuação maior que 22 pontos, indicando que estes idosos apresentavam grande independência funcional para atividades instrumentais de vida diária até um mês antes da internação. A literatura traz que a prevalência de fragilidade e o declínio da funcionalidade aumentam com a idade, ${ }^{27,28}$ sendo maiores em idosos longevos (80 anos de idade ou mais). ${ }^{29}$

A principal causa de internação da amostra estudada foi a investigação de distúrbios gastrointestinais, o que pode ter acontecido devido à exclusão de indivíduos com déficit cognitivo, demência e delirium. A exclusão desses indivíduos pode ser considerada um ponto forte neste estudo, pois permitiu mostrar que, em indivíduos sem déficit cognitivo, existe associação entre a força muscular e capacidade de realizar

\section{REFERÊNCIAS}

1. Ministério da Saúde (Brasil), Secretaria de Atenção à Saúde. Atenção à saúde da pessoa idosa e envelhecimento. Brasília (DF): Ministério da Saúde; 2010.

2. Siqueira AB, Cordeiro RC, Perracini MR, Ramos LR. Impacto funcional da internação hospitalar de pacientes idosos. Rev Saúde Pública 2004;38(5):687-94.

3. Gill TM, Allore HG, Gahbauer EA, Murphy TE. Change in disability after hospitalization or restricted activity in older persons. Journal of American Medical Association 2010;304(17):1919-28.

4. Markle-Reid M, Browne G. Conceptualizations of frailty in relation to older adults. J Adv Nurs 2003;44(1):58-68.

5. Boyd CM, Qian-Li X, Simpson CF, Guralnik JM, Fried LP. Frailty, hospitalization, and progression of disability in a cohort of disabled older women. Am J Med 2005;118(11):1225-31.
AIVDs. Outro ponto forte foi a utilização da medida da força de preensão palmar como desfecho. A força de preensão palmar pode ser considerada indicadora de força muscular global no idoso e foi incluída por Fried et al. ${ }^{30}$ como um dos indicadores de fragilidade nesses indivíduos, podendo ser fácil e rapidamente aplicada ao pacientes idosos na prática clínica.

O curto período de coleta de dados, baixo número amostral e idade maior ou igual a 80 anos na maioria da amostra estudada são considerados fatores limitantes deste trabalho. Contudo, o estabelecimento de critérios de elegibilidade bem definidos aumentou a fidedignidade da amostra final.

\section{CONCLUSÃO}

Este estudo verificou que pessoas idosas sem déficit cognitivo apresentam associação entre a força de preensão palmar e a capacidade de realizar atividades instrumentais de vida diária. Este estudo deve ser continuado, para que a associação destas medidas de funcionalidade seja mais amplamente utilizada na prática clínica para rastreamento de declínio funcional.

6. Szlejf C. Eventos adversos médicos em idosos hospitalizados: frequência e fatores de risco em enfermaria de geriatria [tese]. São Paulo (SP): Universidade de São Paulo, Faculdade de Medicina; 2010. 90 p.

7. Teixeira INDO. Revisão da literatura sobre conceitos e definições de fragilidade em idosos. Rev Bras Promoç Saúde 2008;21(4):297-305.

8. Marin MJS, Bazaglia FC, Massarico AR, Silva CBA, Campos RT, Santos SC. Características sóciodemográficas do atendimento ao idoso após alta hospitalar na estratégia da saúde de família. Rev Esc Enferm USP 2010;44(4):962-8.

9. Kawasaki K, Diogo MJD. Impacto da hospitalização na independência funcional do idoso em tratamento clínico. Acta Fisiatrica 2005;12(2):55-60.

10. Nunes DP, Nakatani AYK, Silveira EA, Bachion MM, Souza MR. Capacidade funcional, condições 
socioeconômicas e de saúde de idosos atendidos por equipes de saúde da família de Goiânia (GO-Brasil). Ciênc Saúde Coletiva 2010;15(6):2888-98.

11. Alves LC, Leite IC, Machado CJ. Fatores associados à incapacidade funcional dos idosos no Brasil: análise multínivel. Rev Saúde Pública 2010;44(3):2-11.

12. Hairi NN, Cumming RG, Naganathan V, Handelsman DJ, Le Couteur DG, Creasey H, et al. Loss of muscle strength, mass (sarcopenia), and quality (specific force) and its relationship with functional limitation and physical disability: the concord Health and ageing in men project. J Am Geriatr Soc 2010;58(11):2055-62.

13. Dorantes-Mendoza G, Avila-Funes JA, Mejía-Arango S, Gutiérrez-Robledo LM. [Factors associated with functional dependence in older adults: a secondary analysis of the National Study on Health and Aging, Mexico, 2001]. Rev Panam Salud Pública 2007;22(1):111. Espanhol.

14. Rovner BW, Casten RJ, Leiby BE. Cognitive and noncognitive determinants of everyday activities in a racially diverse population of older persons receiving health services. J Nerv Ment Dis 2012;200(7):627-31.

15. Novaes RD, Miranda AS, Silva JO, Tavares BVF, Dourado VZ. Equações de referência para a predição da força de preensão manual em brasileiros de meia idade e idosos. Fisioter Pesqui 2009;16(3):217-22.

16. Ling CHY, Taekema D, Craen AJM, Gussekloo J, Westendorp RGJ, Maier AB . Handgrip strength and mortality in the oldest old population: the Leiden 85plus study. Can Med Assoc J 2010;182(5):429-35.

17. Andrade FA, Pereira LV, Sousa FAEF. Mensuração da dor no idoso: uma revisão. Rev Latino-am Enfermagem 2006;14(2):271-6.

18. Burnetto AF, Paulin E, Yamaguti WPS. Comparação entre a escala de Borg modificada e a escala de Borg modificada análogo visual aplicadas em pacientes com dispneia. Rev Bras Fisioter 2002;6(1):41-5.

19. Folstein FF, Folstein SE, McHugh PR. Mini-mental state: a practical method for grading the cognitive states for the clinician. J Psychiatr Res 1975;12(3):189-98.
20. Inouye SK, van Dyck CH, Alessi CA, Balkin S, Siegal AP, Horwitz RI. Clarifying confusion: the confusion assessment method. A new method for detection of delirium. Ann Intern Med 1990;113(12):941-8.

21. Lawton MP, Brody EM. Assessment of older people: self-maintaining and instrumental activities of daily living. Gerontologist 1969 autumn;9(3):179-86.

22. Santos RL, Virtuoso JSJ. Confiabilidade da versão brasileira da escala de atividades instrumentais da vida diária. Rev Bras Promoç Saúde 2008;21(4):290-6.

23. Abdalla IM, Brandão MC. Forças de preensão palmar e da pinça digital. In: Sociedade Brasileira de Terapeutas da Mão. Recomendações para avaliação do membro superior. 2. ed. São Paulo: SBTM; 2005. p.38-41.

24. Ribeiro LHM, Neri AL. Exercícios físicos, força muscular e atividades de vida diária em mulheres idosas. Ciênc Saúde Coletiva 2012;17(8):2169-80.

25. Dutra MM, Muriguchi EH, Lampert MA, Poli-deFigueiredo CE. Validade preditiva de instrumento para identificação do idoso em risco de hospitalização. Rev Saúde Pública 2011;45(1):106-12.

26. Estrella K, Oliveira CEE, Sant'Anna AA, Caldas CP. Detecção do risco para internação hospitalar em população idosa: um estudo a partir da porta de entrada no sistema de saúde suplementar. Cad Saúde Pública 2009;25(3):507-12.

27. Daniels R, Rossum E, Beurskens A, Van den Heuvel W, Witte L. The predictive validity of three selfreport screening instruments for identifying frail older people in the community. BMC Public Health 2012;69(12):1-16.

28. Topinková E. Aging, Disability and Frailty. Ann Nutr Metab 2008;52 suppl 1:6-11.

29. Levers MJ, Estabrooks, CA, Kerr JCR. Factors contributing to frailty: literature review. J Adv Nurs 2006;56(3):282-91.

30. Fried LP, Tangen CM, Walston J, Newman AB, Hirsch C, Gottdiener J, et al. Frailty in older adults: Evidence for a phenotype. J Gerontol 2001;56(3):146-56. 